\title{
Searching New Crystalline Substrates for OMBE: Topological and Energetic Aspects of Cleavable Organic Crystals
}

\author{
Pavel N. Zolotarev, ${ }^{\dagger}$ Massimo Moret, ${ }^{*}+$ Silvia Rizzato, $^{\S}$ and Davide M. Proserpio ${ }^{*} \dagger, \S$ \\ 'Samara Center for Theoretical Materials Science (SCTMS), Samara State Aerospace University, (National Research University) \\ named after academician S.P.Korolyev, SSAU, Moskovskoe Shosse 34, 443086 Samara, Russia \\ ${ }^{\ddagger}$ Dipartimento di Scienza dei Materiali, Università degli Studi di Milano Bicocca, Via R. Cozzi 55, 20125 Milano, Italy \\ ${ }^{\S}$ Dipartimento di Chimica, Università degli Studi di Milano, Via C. Golgi 19, 20133 Milano, Italy
}

Supporting Information

ABSTRACT: Thin films of organic semiconductors are intensively studied in view of their exploitation in modern electronic devices. Until now, few crystalline substrates have been used as clean surfaces for organic molecular beam epitaxy (OMBE). Among them, $\beta$-alanine crystals recently proved to be valuable substrates because of their cleavage along (010) surfaces. To increase the set of available substrates, we searched layered structures through the Cambridge Structural Database by means of the ToposPro program package. Also, we propose here an energy partitioning scheme with a new descriptor that allows one to determine the feasibility of cleaving a crystal along a given crystallographic plane. This procedure is based on the partition of cohesive energy among molecules in a given layer in the crystal structure and molecules found in adjacent layers. The experimental validation on 12 selected amino acid structures, selected among potentially suitable compounds for the OMBE trials, showed the reliability of the method proposed here.

\section{INTRODUCTION}

Organic crystalline materials suitable for production of active layers in optoelectronic devices have been under study for several years. Despite intense efforts, organic electronics has not yet reached large quotas in the mass market, especially for field effect transistors and solar cells. Among many parameters limiting a wider exploitation of organic materials in their crystalline form, crystal structures greatly determine the final physical properties, and hence performances, of organic devices prepared by vacuum deposition techniques or with solution methods. One key parameter is the crystallinity of the thin film layers which can be improved by a proper choice of (crystalline) substrate that can induce the growth of true epitaxial thin film overlayers. ${ }^{1-5}$ Therefore, further study of organic thin films requires a search for new and better behaving crystalline substrates. ${ }^{6,7}$

The list of substrates available as single crystals in organic electronics studies is not very extensive. This includes inorganic systems (mica, alkali halides, oxides) and a few organic crystal phases which can be best suited to trigger heteroepitaxy on large areas and with a few or just one azimuthal orientations of the overlayer. Historically, potassium hydrogen phthalate (KAP) is probably the most studied example with applications ranging from polymer science to organic semiconductors. ${ }^{1,2}$ Recently, new organic crystal substrates have been introduced such as pentaerithrytol (PET) $)^{8}$ and $\beta$-alanine $\left(\beta\right.$-ala), ${ }^{6,7}$ the last being particularly promising. A fundamental feature shared by $\mathrm{KAP}$, PET, $\beta$-ala, and other substrates is easy cleavage along specific planes. This allows preparation of clean surfaces with low roughness for organic molecular beam epitaxy $(\mathrm{OMBE})^{6}$ or hot wall epitaxy (HWE) ${ }^{9}$ under high vacuum conditions. Attempting to find new cleavable crystalline organic substrates, we pursued a systematic approach combining the huge amount of crystallographic information stored in the Cambridge Structure Database ${ }^{10}$ with a rigorous topological analysis and search of specific intermolecular interactions as performed by ToposPro program package ${ }^{11}$ and augmented with PIXEL and AA-CLP calculations, which are a part of the CLP program package. ${ }^{12,13}$ The necessity of objective analysis of topological and energetic features of crystal structures, without any empirical guesses, is evident by recollecting examples of $\mathrm{L}$ lysine hydrochloride dihydrate ${ }^{14}$ and $\alpha$-lactose monohydrate, ${ }^{15}$ where the presence of robust 3D hydrogen-bonded networks deceptively impart mechanical robustness of crystals of both compounds, though the first one has a cleavage plane and the second one has none.

Results arising from such an approach can be of benefit also for other fields, e.g., polymer crystallization. In fact, epitaxial

Received: November 30, 2015

Revised: February 1, 2016

Published: February 9, 2016 


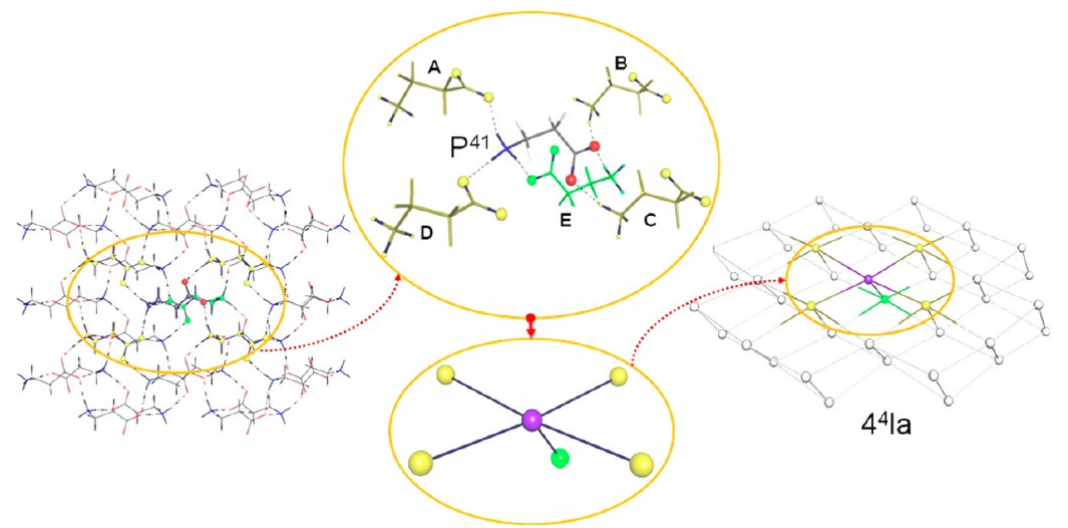

Figure 1. Simplification procedure and resulting underlying net observed for the crystal structure of $\beta$-alanine (BALNIN01). ${ }^{29}$ Left to right: fragment of the hydrogen-bonded double layer; each molecule in the structure has five atoms that participate in HB (three ammonia hydrogens and two carboxylate oxygens). The reference molecule is hydrogen-bonded to five other: to four molecules by one HB bond each (highlighted in yellow, A-D) and to one more by two HBs (highlighted in green, E); therefore, MCTS is $\mathrm{P}^{41}$ and in total there are $4 \times 1+1 \times 2=6$ hydrogen bonds. On the right is the 5 -coordinated $4^{4}$ Ia underlying net.

crystallization of polymers onto organic crystal surfaces is a long-standing area of research ${ }^{16-18}$ which continues in the present day due to the never-ending technological and economical relevance of polymers. ${ }^{19-21}$

Last but not least, mechanical properties of molecular crystals emerging from the topological analysis of crystal structures and their subsequent experimental study are also highly relevant for the pharmaceutical field as tableting. ${ }^{22}$

In the present paper we propose a method to analyze intermolecular interactions in molecular solids with the goal of selecting from the crystallographic databases a set of structures fulfilling the structural requirements for robust and cleavable crystals, to be employed in OMBE or equivalent studies. In this study we restricted our search to amino acids due to the presence of two-dimensional $\mathrm{H}$-bond networks producing highly anisotropic crystal structures. Extraction of potential candidates from the crystallographic databases was performed with the program ToposPro. To reduce biases and subjective judgment during the choice of suitable structures, we introduce an energy criterion-the $X$ parameter-based on partitioning of crystal energies and evaluation of the anisotropy of intermolecular interactions. The analysis of intermolecular energies and their partitioning to find likely cleavage planes was based on PIXEL calculations of the candidate structures. A selection of structures with high $X$ values, i.e., showing highly anisotropic packing interactions and suggesting a probable ease of cleavage, were experimentally checked by growing the corresponding crystals to verify the presence of the predicted cleavage planes. Interestingly, during cleavage of candidate crystals we observed that several crystalline materials exhibit extraordinary plastic deformations apparently in contrast to the robustness of the structural 2D hydrogen-bonded layers involved in the cleavage process.

\section{EXPERIMENTAL SECTION}

Topological Analysis of the Structures. A topological analysis of crystal structures was performed by a procedure described in detail elsewhere and implemented in the ToposPro program package. ${ }^{11}$ All intra- and intermolecular interactions were determined using the Sectors method ${ }^{23}$ in the atomic Voronoi polyhedra approach. Hydrogen bonds $(\mathrm{HB})$ in a fragment $\mathrm{A}-\mathrm{H} \cdots \mathrm{B}$ were identified in accordance with the following additional geometrical criteria: $d(\mathrm{H} \cdots \mathrm{B})$ $\leq 2.5 \AA \AA d(\mathrm{~A} \cdots \mathrm{B}) \leq 3.5 \AA$; $\angle \mathrm{A}-\mathrm{H} \cdots \mathrm{B} \geq 120^{\circ}(\mathrm{A}=\mathrm{N}, \mathrm{O} ; \mathrm{B}=\mathrm{N}, \mathrm{O}, \mathrm{F}$, $\mathrm{S}, \mathrm{Cl}) .^{24}$
The topological analysis performed with program ToposPro provides an appropriate way of obtaining the underlying net for each crystal structure, i.e., a representation of the molecular network in terms of a graph-theory approach. The simplification procedure consists of representing the molecule by its center of mass, keeping the connectivity of the molecule with its neighbors; all intermolecular contacts between a given pair of molecules transform to the same edge between the molecular centers of mass in the simplified net. The resulting underlying net describes the patterns of the intermolecular connections in the whole crystal.

After obtaining the underlying net for a given crystal structure, we determine its topological type by comparing sets of topological indices that unambiguously determine the net. In this work, we use threeletter symbols of the Reticular Chemistry Structure Resource (RCSR) notation $^{25}$ or Fischer and Koch's symbols for 1- or 2-periodic sphere packings. ${ }^{26}$ Those nets that are absent in the RCSR are designated with the Topos $N D n$ nomenclature, ${ }^{27}$ where $N$ is a sequence of coordination numbers of all nonequivalent nodes of the net, $D$ is periodicity of the net $(D=\mathrm{M}, \mathrm{C}, \mathrm{L}, \mathrm{T}$ for 0 -, 1-, 2-, 3-periodic nets, respectively), and $n$ is the ordinal number of the net in the set of all non-isomorphic nets with the given ND sequence.

To characterize the local mutual arrangement of molecules in the structures, we used the notation that was proposed for description of hydrogen-bonded molecules. ${ }^{28}$ Each molecule $(\mathrm{L})$ is designated by letters $\mathrm{M}, \mathrm{B}, \mathrm{T}, \mathrm{K}, \mathrm{P}, \mathrm{G}, \mathrm{H}, \mathrm{O}, \mathrm{N}, \mathrm{D}$ depending on the number $n=1-$ 10 of its atoms (both donors and acceptors) involved in the formation of intermolecular hydrogen bonds. The total number of molecules connected to a reference one is listed as the upper index in the form mbtkpghond..., where each integer $m, b, t, k, \ldots$ is equal to the number of molecules connected by one, two, three, four, ... hydrogen bonds. Ultimately, the molecular connection type symbol (MCTS) looks like $L^{\text {mbktpghond }}$ (Figure 1).

Screening and Selection of Crystal Structures. In order to find potential crystalline substrates for heteroepitaxial growth of organic thin films, we screened the Cambridge Structural Database (v 5.36 February 2015) by means of the ToposPro package. In this first study, we looked for crystal structures of amino acids or their derivatives with the ability to cleave neatly along specific crystallographic planes. Potential candidates had to be cheap, commercially available, and nontoxic compounds, exhibiting high or significant solubility in water or nontoxic solvents and possibly giving millimeter-sized crystals. The absence of any solvates and hydrates for the searched structures was mandatory to guarantee stability to ultrahigh-vacuum conditions needed for OMBE experiments. $\beta$-Alanine crystals, recently used with success for organic-organic heteroepitaxy, ${ }^{6,7}$ are easily obtained as centimeter-sized individuals cleaving parallel to (010) planes owing to a 2-periodic hydrogen-bonded network. To set up a general and exhaustive procedure for searching new suitable crystal substrates 
exploiting the wealth of information stored in the crystallographic databases, instead of relying upon single and serendipitous findings, we have developed an approach based on a search of structures followed by a topological analysis depicted on Scheme 1. As result, from 199

Scheme 1. Steps of Data Retrieval and Further Computations for Structure Selection ${ }^{a}$

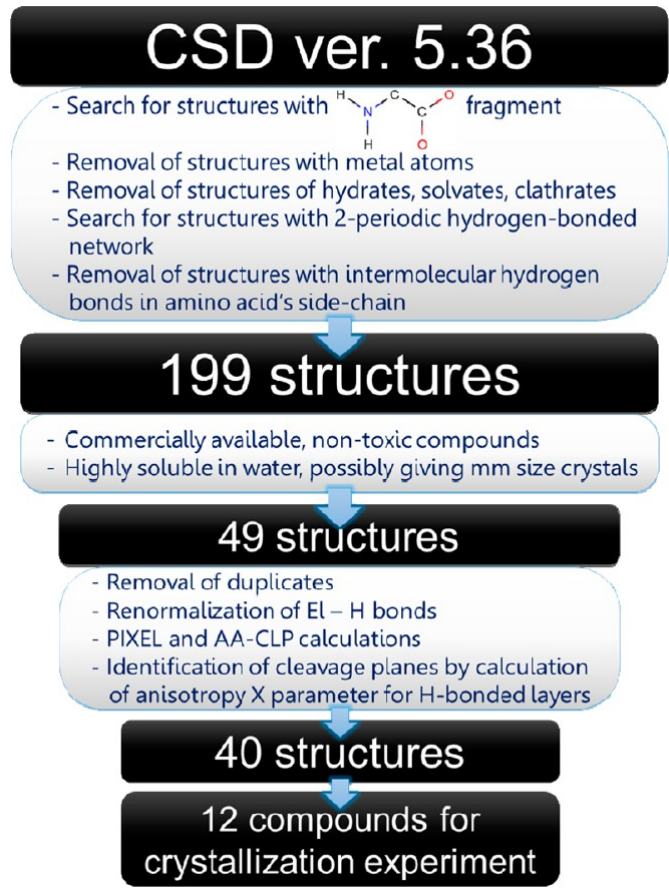

${ }^{a}$ For definition of the $X$ parameter see Descriptor of intermolecular interactions anisotropy.

structures we computed 40 structures and out of these we selected 12 candidates based on the presence of accurate studies, all by $\mathrm{C}$. $\mathrm{H}$. Görbitz in the present case, as a test of the performance of the proposed method.

Computational Methods. Lattice energies as well as intermolecular interaction energies (IIE), partitioned in electrostatic, polarization, dispersion, and repulsion terms, were calculated by means of the PIXEL method ${ }^{12}$ and atom-atom molecular force field AA-CLP ${ }^{13}$ calculations both implemented in the CLP package. The procedure requires normalization of bond distances involving $\mathrm{H}$ atoms to values typically obtained from neutron diffraction experiments, i.e., $\mathrm{C}-\mathrm{H}$ to $1.08 \AA, \mathrm{O}-\mathrm{H}$ and $\mathrm{N}-\mathrm{H}$ to $1.00 \AA$, and quaternary ammonium $\mathrm{N}-\mathrm{H}$ to $1.03 \AA$. Molecular electron densities required as input files for PIXEL were calculated by means of ab initio methods implemented in the Gaussian $09^{30}$ package at the MP2/6-31G(d,p) level of theory. As shown in ref 31 the results of PIXEL calculations, namely, lattice energies and cohesive energies of molecular dimers extracted out of a crystal, are in overall agreement with other ab initio computational schemes. Standard condensation level $n=5$ was used in calculations. The influence of the condensation level on the computed values of $X$ parameter (see later) was checked on the DL-norleucine structure (DLNLUA02) and revealed only small deviations, namely, $X(100)=$ 0.940 at $n=4,0.943$ at $n=5$, and 0.941 at $n=6$.

Crystallization Experiments. As an experimental confirmation for the presence of crystallographic planes suitable for easy cleavage, a series of crystallization experiments were conducted on 12 species selected from a list of 40 structures previously subjected to a thorough computational analysis. Aqueous solutions were prepared by dissolving pure amino acids or equimolar mixtures of two amino acids in deionized water. The solutions were poured into test-tubes which were subsequently placed into a thermostated bath whose temperature was kept at $30 \pm 0.05{ }^{\circ} \mathrm{C}$. Crystals of typical size $1-7 \mathrm{~mm}$ appeared as isopropanol vapors diffused into aqueous solutions. DL-Phenylglycine crystals were grown from hot saturated aqueous solutions by slow cooling.

X-ray Crystallographic Studies. The phase nature of all 12 aforementioned samples was checked by using single crystal X-ray diffraction to determine the unit cell dimensions and the crystal symmetry to compare with the lattice parameters of the known structures. All measurements were carried out at ambient temperature on a Bruker APEX II CCD area-detector diffractometer performing a short data collection of three sets of exposure frames starting from different $\varphi$ angles, each covering $30^{\circ}$ of rotation in $\omega$, with a scan step of $0.3^{\circ}$ and an exposure time per frame ranging from 10 to $20 \mathrm{~s}$.

A crystallographic description of the crystals' morphologies has been obtained by indexing the relevant faces of the crystals and reconstructing the image of the crystals' shapes using the Face Indexing Module in the Apex 2 software. ${ }^{32}$

Test of Mechanical Properties. With the aim to check the presence of cleavage planes in the grown crystals we used the following procedure: the crystal lying on the worktop of a microscope was subjected to the mild pressure of a sharp razor blade onto available crystal faces. If the crystal split easily along the expected plane, i.e., without the appearance of additional fractures and showing a smooth cleaved surface, we assumed that the crystal has positively passed the test and a perfect cleavage plane does exist parallel to the selected $\{h k l\}$ family of planes. When a crystal is too soft and undergoes a plastic deformation under stress (e.g., bending deformation) we used sticky tape to verify the presence of a cleavage plane by stripping thin flakes of the crystal.

\section{RESULTS AND DISCUSSION}

Distributions of Topological Parameters. The hydrogen-bonded underlying nets found in all 199 extracted structures with side-chains not involved in intermolecular $\mathrm{H}$ bonding are listed in the Supporting Information (Figure S1, Table S1). Analyzing this distribution, we see that the observed nets can be divided into two types: 2-periodic 3D thick layers ( $\left.4^{4} \mathrm{Ia}, 4^{4} \mathrm{IIb}, 5 \mathrm{~L} 7,4^{4} \mathrm{IV}, 6 \mathrm{~L} 13,6 \mathrm{~L} 3,4,4 \mathrm{~L} 27\right)$, which prevail in this class of compounds and cover $84 \%$ of structures, and less abundant 2-periodic 2D simple layers (sql, hcb, hxl, cem, tts). The analysis of the structures revealed that the nomenclature proposed in ref 33 for the description of hydrogen-bonded nets in amino acids can be fully rationalized and extended using the two topological descriptors such as the MCTS symbol and topological type of underlying net (Figure S2, Table S2, and the spreadsheet Table X1 in the Supporting Information). For example, for LD-LD sheets we found connection types of molecules $\mathrm{P}^{6}$ and $4^{4} \mathrm{IIb}$ net topology. L2-L2 sheets in fact might be divided in two subclasses: structures with $5 \mathrm{~L} 7$ net and MCTS P, ${ }^{32}$ e.g., LEUCIN02, and structures with 6L13 net and MCTS of molecules $\mathrm{P}^{42}$ e.g., LVALIN01. This example shows that the use of topological descriptors paves the way for a rigorous and more thorough description of hydrogen-bonded networks and patterns, at least for the structures with precisely localized hydrogen positions. Since the quality of the structures extracted from crystallographic databases can vary significantly, especially concerning the $\mathrm{H}$ atom positions, the use of quantum-chemical methods to "improve" the structure quality may be of great help. ${ }^{34}$

Descriptor of Intermolecular Interactions Anisotropy. It is a well-established fact that hydrogen bonds are among the strongest intermolecular interactions in organic solids ${ }^{35}$ and that one of the techniques for identifying cleavage planes, besides attachment energy calculations, "...is visualization of crystal structure, where the rigid crystallographic planes (often strengthened by hydrogen bonds within the planes) with 


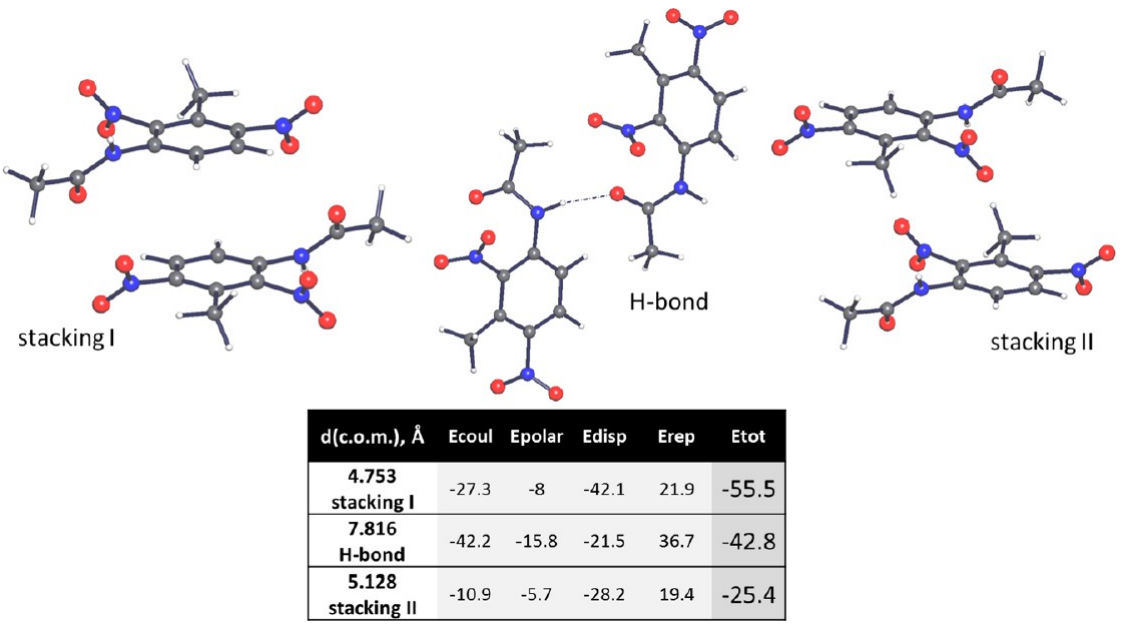

Figure 2. Molecular pairs in the structure of 3-(acetamido)-2,6-dinitrotoluene (YAHJUN). Stacking I between molecules at $4.753 \AA$ surpasses in interaction energy the hydrogen bonded molecular pair at 7.816 ̊. Slightly shifted position of molecules in stacking II pair leads to almost 2 -fold decrease in total interaction energy compared to stacking I pair.

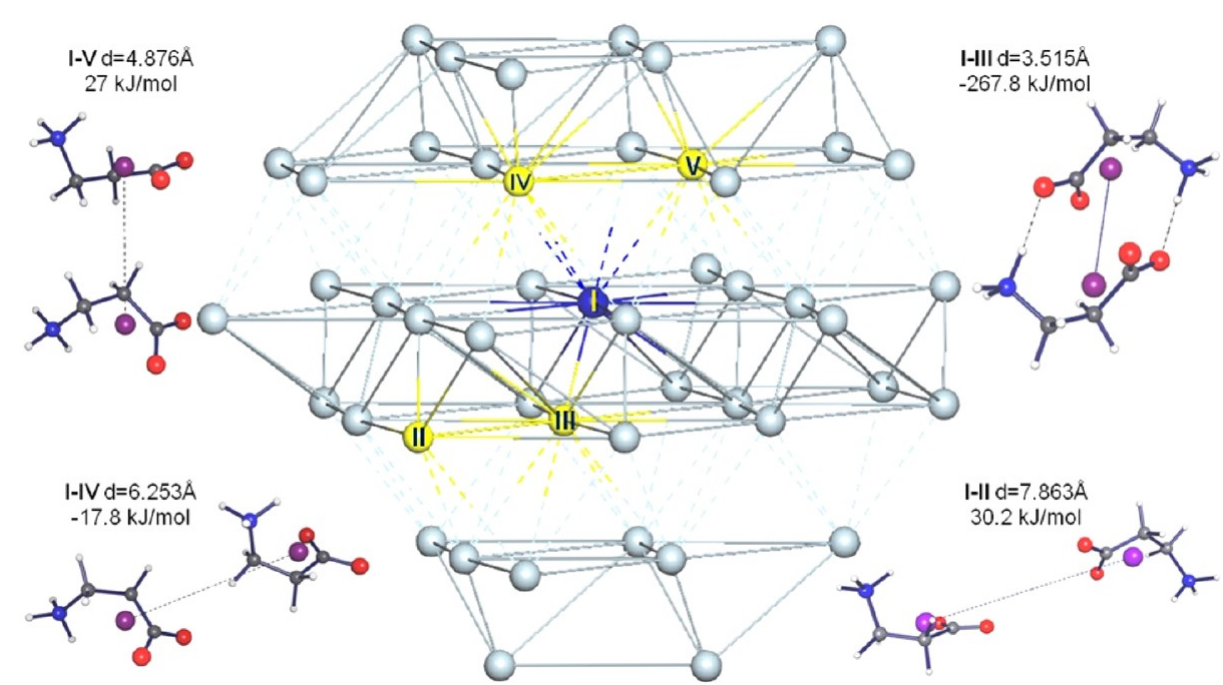

Figure 3. Picture of the cluster of $\beta$-alanine molecules (spheres represent centers of mass of molecules) generated by symmetry elements around the central molecule (in blue). Solid lines connect molecules in the same hydrogen-bonded layer; contacts between molecules in adjacent layers are shown as dashed lines. Molecular pairs from the first (I-III, I-IV and I-V) and the second (I-II) coordination spheres of the central molecule are shown alongside the distances between centers of mass and values of intermolecular interaction energies. Note the destabilizing contact I-II with quite high interaction energy of $30 \mathrm{~kJ} / \mathrm{mol}$ despite the long distance.

highest molecular density and largest $d$-spacing are the slip or cleavage planes". ${ }^{36}$ So, intrinsically, the idea to take into account the strength of intermolecular interactions, primarily hydrogen bonds, was already in use. However, we have to keep in mind that the strength of conventional hydrogen bonds varies significantly, e.g., from mostly electrostatic in nature in water or alcohols with typical energy $15-60 \mathrm{~kJ} / \mathrm{mol}$ to partly covalent strong charge-assisted $\mathrm{H}$-bonds in amino acids zwitterions with interaction energies in the range $60-180 \mathrm{~kJ} /$ mol. $^{37}$ A second major point is that other interactions, sometimes nonspecific and hardly identifiable on a geometrical basis, between pertinent regions of the molecule can compete in strength with H-bonds, as for example, in 3-(acetamido)-2,6dinitrotoluene (YAHJUN), ${ }^{38}$ where there is an intermolecular contact that is comparable in total interaction energy with $\mathrm{NH} \cdots \mathrm{O}$ hydrogen bond (Figure 2). The reason lies in the predominance of the Coulombic-polarization term over the dispersion term in the $\mathrm{H}$-bonded dimer, though short interatomic distance resulted in a higher repulsion term that lowers the overall stabilization, which is consistent with the analysis of crystal structures reported in ref 39.

Therefore, a nonbiased choice of cleavage or slip planes should not rely on very subjective visual methods for their identification. In order to add a more quantitative analysis of crystal structures and find a measure of the propensity of molecular crystals to cleave along distinct crystallographic planes we propose an energy-based scheme exploiting the estimation of intermolecular interaction energies (IIE) anisotropy in crystal structure. The model crystal is represented by a spherical cluster of molecules generated from a central reference molecule, according to the pertinent space group symmetry. Molecules in the cluster are all those within a given cutoff distance (usually, 15 to $20 \AA$ is enough to take into account molecules in the first and second coordination shell of the reference molecule) between molecular centers of mass. 
Division of the structure into adjacent layers allows one to compute the $X$ parameter for a given layer as follows:

$$
X=\frac{\sum_{i} \text { IIE reference molecular to } i \text { th layer molecule }}{\sum_{j} \text { IIE reference molecule to } j \text { th cluster molecule }}
$$

where $i$ is the number of molecules in the reference layer to which the central molecule belongs; $j$ is the number of molecules in the cluster generated by PIXEL or AA-CLP procedures for calculation of IIE values. The physical meaning of the $X$ parameter is related to IIE anisotropy, that is, the share of cohesive energy in the layer compared to the total cohesive energy in the cluster. In the $\beta$-alanine example shown in Figure 3 we have to sum up 23 IIE central molecule-molecules in the same hydrogen-bonded layer terms in the numerator. For the denominator we have to sum up $23+24$ IIE central molecule-all molecules in the spherical cluster; we did not restrict the summation to the first coordination shell because one can find quite strong interactions, especially in crystals with zwitterions and molecules with highly polar functional groups. The present approach is similar to the concept of dimensional analysis proposed in refs ${ }^{40-42}$ where the total lattice energy was also partitioned into components belonging to layers, chains, and molecular dimers, though in those studies DFT-D calculations were applied.

Opposite to the purely geometrical analysis of crystal packing, the development of computational methods gave an opportunity to resolve the problems of hierarchy and of relative significance in intermolecular bonding. ${ }^{39,43}$ Energy-based description of crystal structures is now an emergent standpoint that offers more objective consideration of the crucial interactions and recognition of all levels of organization of molecular crystals. ${ }^{44}$ In ref 45 , the interrelation of mechanical properties of molecular solids and their "energy frameworks" was pointed out. A couple of studies were devoted to elucidation of the perfection degree of the cleavage planes ${ }^{46,47}$ of potassium dichromate crystal. The authors proposed two parameters, related to the surface energy of the $h k l$ plane, responsible for the smoothness of the freshly cleaved surface of the crystal.

After calculating the $X$ values for a set of layers in a crystal structure, we assume that the crystal is prone to split along the plane with the highest $X$ value. We restricted the $X$ value calculation to the plane comprising the H-bonded layers and other planes (usually those having small Miller indexes) reasonably cutting the crystal structure and resulting in high intralayer energies. A future version of our procedure will implement an automatic vectorial search of the planes in the structure. For example, $\beta$-alanine has hydrogen-bonded double layers in the $(010)$ plane. The $\beta$-alanine crystal structure can thus be imagined as built by double layers held together by an extended network of $\mathrm{H}$-bonds. The resulting hydrophobic bilayers interact weakly due to dispersion forces, which produce a low value of the surface energy responsible for the perfect cleavage without disruption of the $\mathrm{HB}$ bilayers. ${ }^{48}$ For other planes in the structure we obtain the values reported in Figure 4.

As a validation of our calculations, the squeezing of a large flat crystal of $\beta$-alanine between two glass plates showed the emergence of secondary cleavage planes. As can be seen in the sequence of pictures on Figure 5, already in the first stages of compression small fractures parallel to $\{111\}$ planes appear in the crystal, which further enlarge as the applied stress rises. This
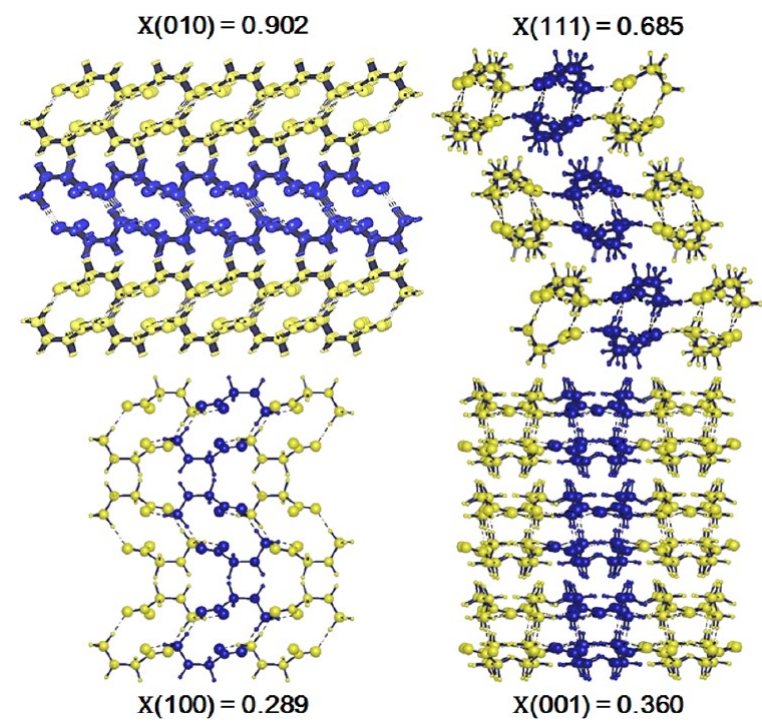

Figure 4. Different sets of adjacent planes in $\beta$-alanine structure are colored blue and yellow. For each plane the calculated value of $X$ is shown. During the cleavage, disruption of the hydrogen-bonded network occurs in all cases except for the (010) plane.

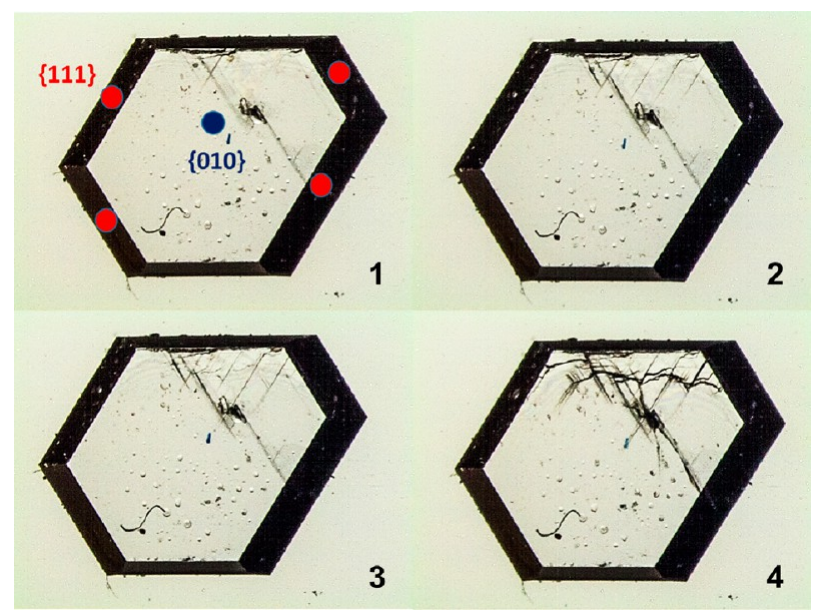

Figure 5. Sequential frames of a $\beta$-alanine crystal squeezed between two glass plates. Cleavage fractures parallel to $\{111\}$ develop with increasing load on the $\{010\}$ faces.

is in accordance with ranking of $\{h k l\}$ planes based on the $X$ parameter, $X(111)=0.685$ ranking second after $X(010)=$ 0.902 but still significant.

Cleavage Plane of the Crystals. Among the 12 crystals selected on the basis of their relevance in previous studies, ${ }^{49-55}$ only one co-crystal, namely, L-Leu/D-Met, gave small polycrystalline aggregates of tiny needles or long hollow tubes not suitable for mechanical testing. Large and wellformed plate-like crystals were obtained for co-crystals L-Val/DNval, L-Val/D-Met, L-Ile/D-Nval, and L-Ile/D-Met. Crystals of LPhe/D-Nval and L-Phe/D-Met appeared as long needles. L-Met, DL-Val, DL-Ile, and co-crystal L-Ile/D-Ala gave thin and mostly twinned crystals of bad quality. A summary on the crystallization experiments is presented in Table 1.

The crystals obtained can be easily split along a cleavage plane. In all cases, the cleavage plane has been identified by indexation of corresponding crystal faces and coincides with the layer that contains the hydrogen-bonded network and has high 
Table 1. Results of Crystallization Trials and Tests of Cleavage Properties ${ }^{a}$

\begin{tabular}{|c|c|c|c|c|c|c|c|c|c|c|}
\hline \multirow[b]{2}{*}{ refcode } & \multirow[b]{2}{*}{ name } & \multirow[b]{2}{*}{$\begin{array}{l}\text { space } \\
\text { group }\end{array}$} & \multicolumn{6}{|c|}{ HB network } & \multirow[b]{2}{*}{$\begin{array}{l}\text { cleavage } \\
\text { plane }\end{array}$} & \multirow[b]{2}{*}{ crystal habit } \\
\hline & & & topology & MCTS & $\begin{array}{l}\text { Görbitz } \\
\text { notation }\end{array}$ & plane & $\begin{array}{c}X \\
(\mathrm{AA}-\mathrm{CLP})\end{array}$ & $\begin{array}{c}X \\
\text { (PIXEL) }\end{array}$ & & \\
\hline DLILEU02 & DL-Isoleucine & $P \overline{1}$ & $4^{4} \mathrm{Ia}$ & $\mathrm{P}^{41}$ & L1-D1 & $(001)$ & 0.927 & 0.967 & $(001)$ & $\begin{array}{l}\text { thin plates } 3-7 \mathrm{~mm} \text {, mostly } \\
\text { twinned }\end{array}$ \\
\hline VALIDL02 & DL-Valine & $P \overline{1}$ & $4^{4} \mathrm{Ia}$ & $\mathrm{P}^{41}$ & L1-D1 & $(001)$ & 0.845 & 0.949 & $(001)$ & $\begin{array}{l}\text { flakes/thin plates } 1-4 \mathrm{~mm} \text {, } \\
\text { mostly twinned }\end{array}$ \\
\hline BERNIV & $\begin{array}{l}\text { L-Leucine } \\
\text { D-Methionine }\end{array}$ & $P 2_{1}$ & $4^{4} \mathrm{Ia}$ & $\mathrm{P}^{41}$ & L1-D1 & $(010)$ & 0.899 & 0.931 & - & $\begin{array}{l}\text { tiny hollow tubes, small } \\
\text { hedgehogs }\end{array}$ \\
\hline FIVGEW & DL-Phenylglycine & $P 2_{1} / c$ & $4^{4} \mathrm{IIb}$ & $\mathrm{P}^{6}$ & LD-LD & $(100)$ & 0.951 & 0.943 & $(100)$ & thick small plates $1-3 \mathrm{~mm}$ \\
\hline FITHIZ & $\begin{array}{l}\text { L-Isoleucine } \\
\text { D-Alanine }\end{array}$ & $P 2_{1}$ & $4^{4} \mathrm{IIb}$ & $\mathrm{P}^{6}$ & LD-LD & $(001)$ & 0.944 & 0.981 & $(001)$ & thin plates, mostly twinned \\
\hline BERQEU & L-Valine D-Norvaline & $\mathrm{C} 2$ & $4^{4} \mathrm{IIb}$ & $\mathrm{P}^{6}$ & LD-LD & $(100)$ & 0.940 & 0.950 & $(100)$ & plates $2-7 \mathrm{~mm}$ \\
\hline FITJEX & $\begin{array}{l}\text { L-Isoleucin } \\
\text { D-Norvaline }\end{array}$ & $\mathrm{C} 2$ & $4^{4} \mathrm{IIb}$ & $\mathrm{P}^{6}$ & LD-LD & $(100)$ & 0.937 & 0.966 & $(100)$ & plates $2-9 \mathrm{~mm}$ \\
\hline FITLID & $\begin{array}{l}\text { L-Isoleucine } \\
\text { D-Methionine }\end{array}$ & $\mathrm{C} 2$ & $4^{4} \mathrm{IIb}$ & $\mathrm{P}^{6}$ & LD-LD & $(100)$ & 0.934 & 0.944 & $(100)$ & plates $2-12 \mathrm{~mm}$ \\
\hline POVYIJ & $\begin{array}{l}\text { L-Phenylalanine } \\
\text { D-Norvaline }\end{array}$ & $P 2_{1} 2_{1} 2_{1}$ & $4^{4} \mathrm{IIb}$ & $\mathrm{P}^{6}$ & LD-LD & $(001)$ & 0.928 & 0.988 & $(001)$ & needles $2-7 \mathrm{~mm}$; rosettes \\
\hline POVYOP & $\begin{array}{l}\text { L-Phenylalanine } \\
\text { D-Methionine }\end{array}$ & $P 2_{1}$ & $4^{4} \mathrm{IIb}$ & $\mathrm{P}^{6}$ & LD-LD & $(001)$ & 0.926 & 0.928 & $(001)$ & needles $3-20 \mathrm{~mm}$; rosettes \\
\hline BERQIY & $\begin{array}{l}\text { L-Valine } \\
\text { D-Methionine }\end{array}$ & $P 2_{1} 2_{1} 2_{1}$ & $4^{4} \mathrm{IIb}$ & $\mathrm{P}^{6}$ & LD-LD & $(100)$ & 0.899 & 0.965 & $(100)$ & plates $2-9 \mathrm{~mm}$ \\
\hline LMETONO2 & L-Methionine. & $P 2_{1}$ & $5 \mathrm{~L} 7$ & $\mathrm{P}^{32}$ & L2-L2 & $(001)$ & 0.968 & 0.976 & $(001)$ & $\begin{array}{l}\text { thin large plates up to } 30 \mathrm{~mm} \text {, } \\
\text { twinned }\end{array}$ \\
\hline
\end{tabular}

${ }^{a}$ Experimentally observed cleavage planes were confirmed by face-indexing procedures with single crystal X-ray diffraction.

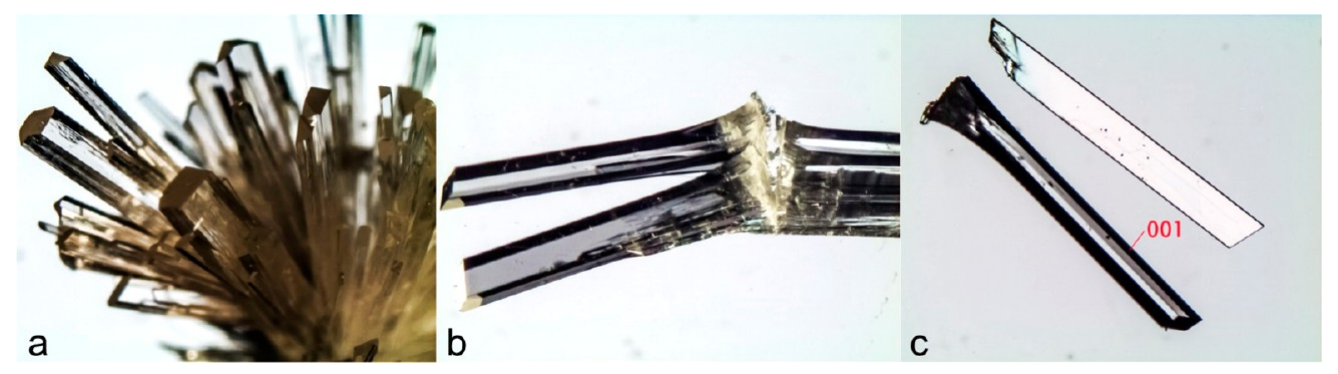

Figure 6. (a) Rosette of L-Phe/D-Met co-crystals grown on the bottom of a test tube; (b) two crystals cut from the rosette; (c) on the upper right, a thin crystal plate separated by cleaving from the original crystal giving a flat and clean surface.

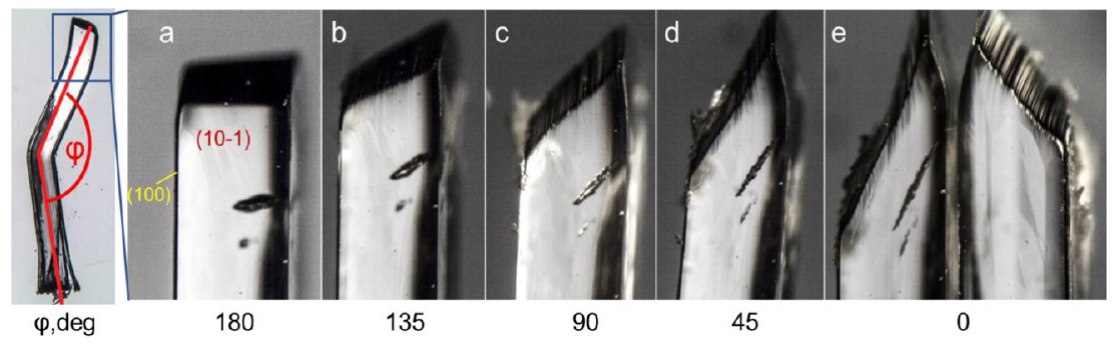

Figure 7. Sequential frames of the change of interfacial angle in L-Val/D-Met co-crystal during the bending of the crystal. In picture (e) one can observe different degrees of interfacial angle changes on the opposite sides of the crystal.

values of the $X$ parameter. For example, L-Phe/D-Met crystals split easily along the (001) plane (Figure 6). Similar results were found for all tested crystals. The results of the calculations of $X$ values for hydrogen-bonded layers in 40 compounds (Scheme 1) are shown in the spreadsheet Table X2 (Supporting Information), providing a starting set for growing large crystals to be used as substrates. The $X$ value for $\mathrm{H}$ bonded layers, showing, as expected, very strong intralayer bonds, establish high anisotropy of intermolecular interactions and imply high probability of being the primary cleavage planes of those crystals.
Crystal Bending. It was somewhat unexpected to find that the investigated crystals can undergo a plastic deformation, namely, bending, without loss of integrity as a whole. Recently, such an unusual mechanical behavior was reported for amino acid salt L-isoleucinium hydromaleate hemihydrate, which also has a $2 \mathrm{D}$ hydrogen-bonded system. ${ }^{56}$ In the present investigation, the bending faces and cleavage faces were found to be the same in all the crystals studied, i.e., the hydrogen-bonded layers can either exfoliate or bend and slide on top of each other as evidenced by considerable change in the interfacial angles (i.e., angle between crystal faces) of the $\mathrm{L}-\mathrm{Val} /$ D-Met crystal (Figure 7). 

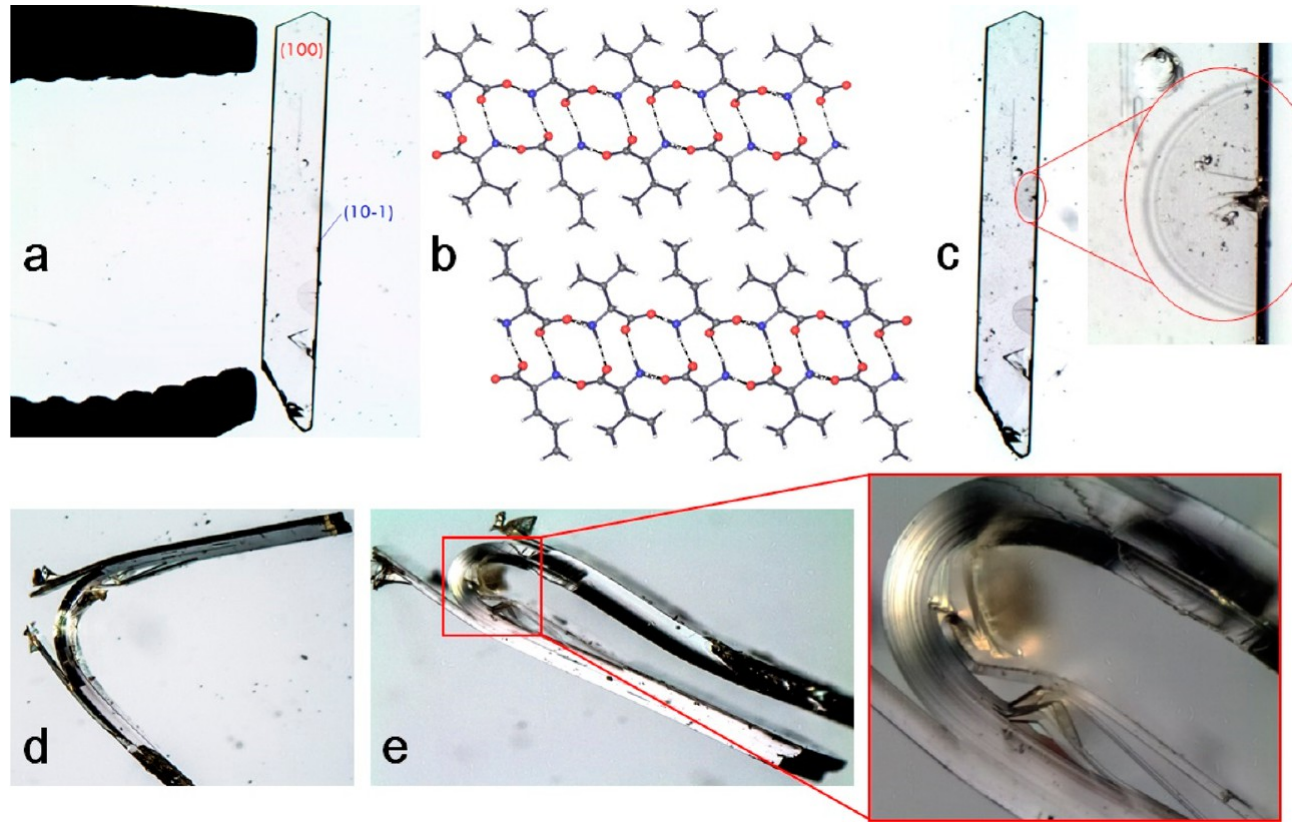

Figure 8. (a) Crystal of L-Val/D-Nval with its (100) and (10̄) faces. The size is about $7 \mathrm{~mm}$. (b) Hydrogen-bonded layers in the (100) plane in the structure of L-Val/D-Nval; (c) application of stress perpendicular to (10) $)$ face leads to cracking together with cleavage parallel to (100) as evidenced by the semicircular wedge centered on the impact point; $(d, e)$ crystal easily bends on the (100) face; striation pattern is visible on the side face.
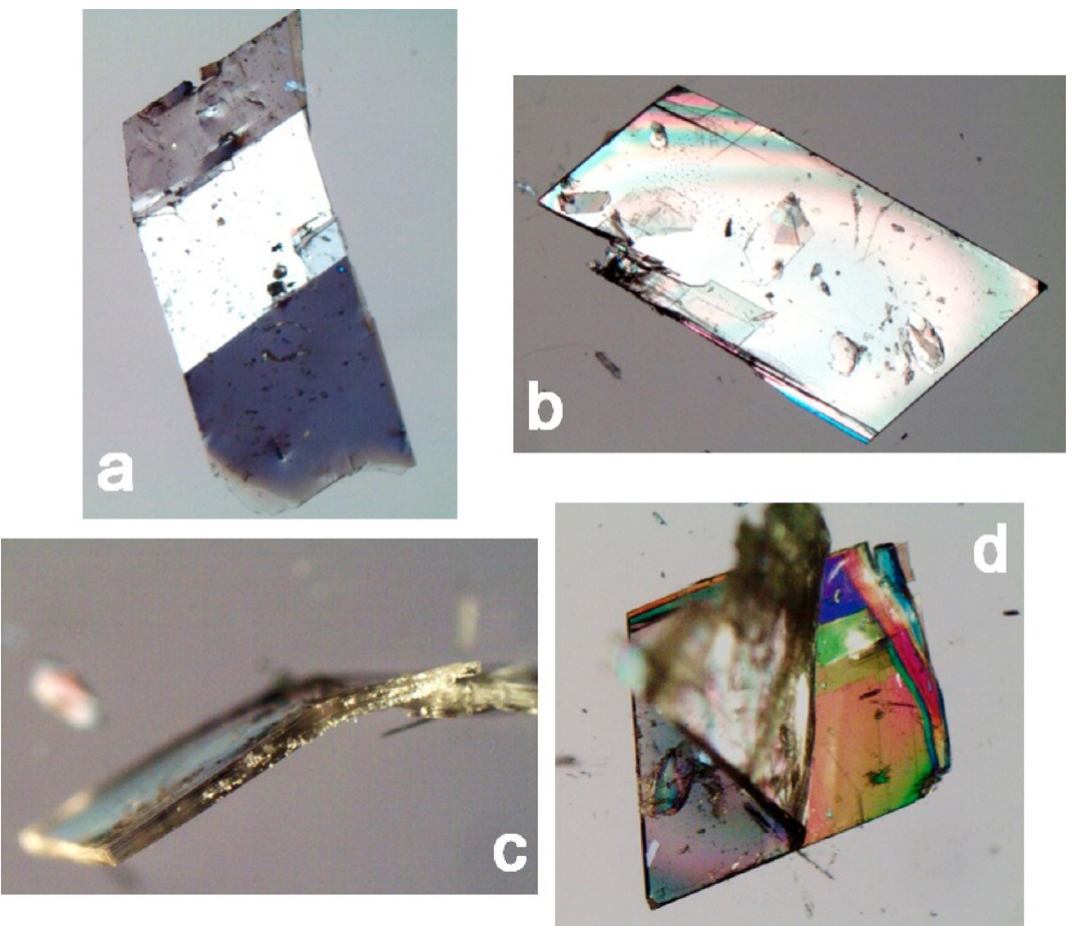

Figure 9. (a) Thin plate of DL-valine crystal breaks when trying to bend it; (b,c,d) thicker crystal is bendable on the (001) face, though multiple cracks occur while arching.

The bending of the molecular crystals may resemble the bending of a pile of paper sheets, where sheets represent single hydrogen-bonded layers. Initially this pile has right angles between its edges, but when it is curved the interfacial angles change, since almost undeformed and unstretched layers slide on top of each other. Trials to bend the crystals by applying pressure on other faces lead to fracture, highlighting the strong anisotropy of bending capability (Figure 8).
The capability of bending has been found in all the investigated crystals, though in most cases bending was accompanied by cleavage that may be due to crystal imperfections. As for DL-valine crystals, they seem to be more rigid and brittle compared to others. Thin plates are brittle; thicker ones crack during the bending (Figure 9).

Regarding the origin of such seemingly unusual mechanical feature, several explanations can be found in the literature. In a study on structure-property correlations in bending and brittle 

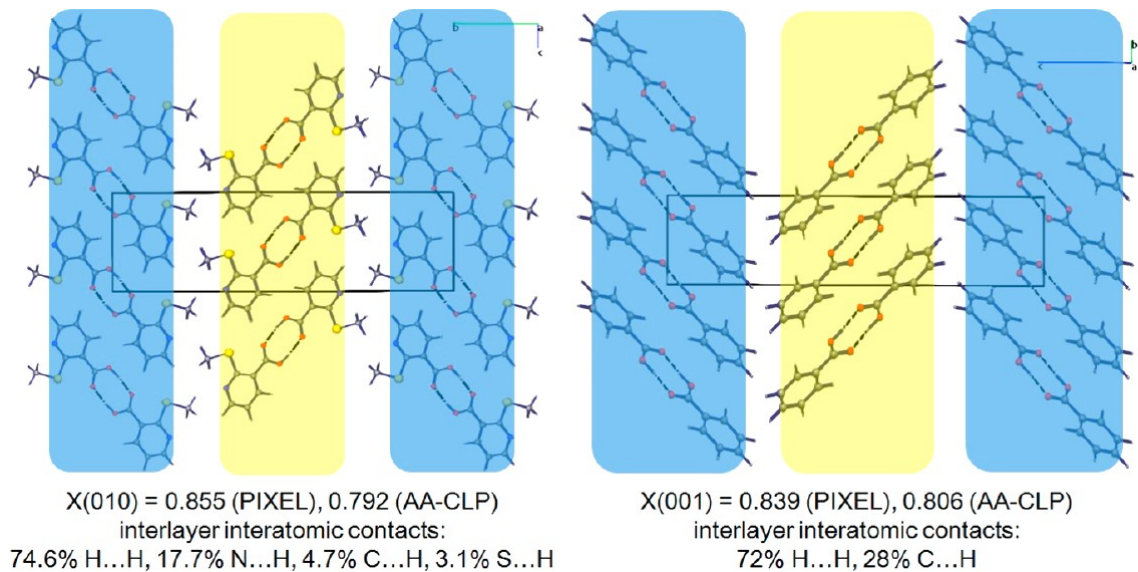

$72 \% \mathrm{H} . . . \mathrm{H}, 28 \% \mathrm{C} . . . \mathrm{H}$

Figure 10. Fragment of the crystal structures of 2-methyltionicotinic acid (KAMQIZ) (left) and benzoic acid (BENZAC12) (right). The shares of interlayer interatomic contacts were calculated using Voronoi-Dirichlet polyhedra approach.

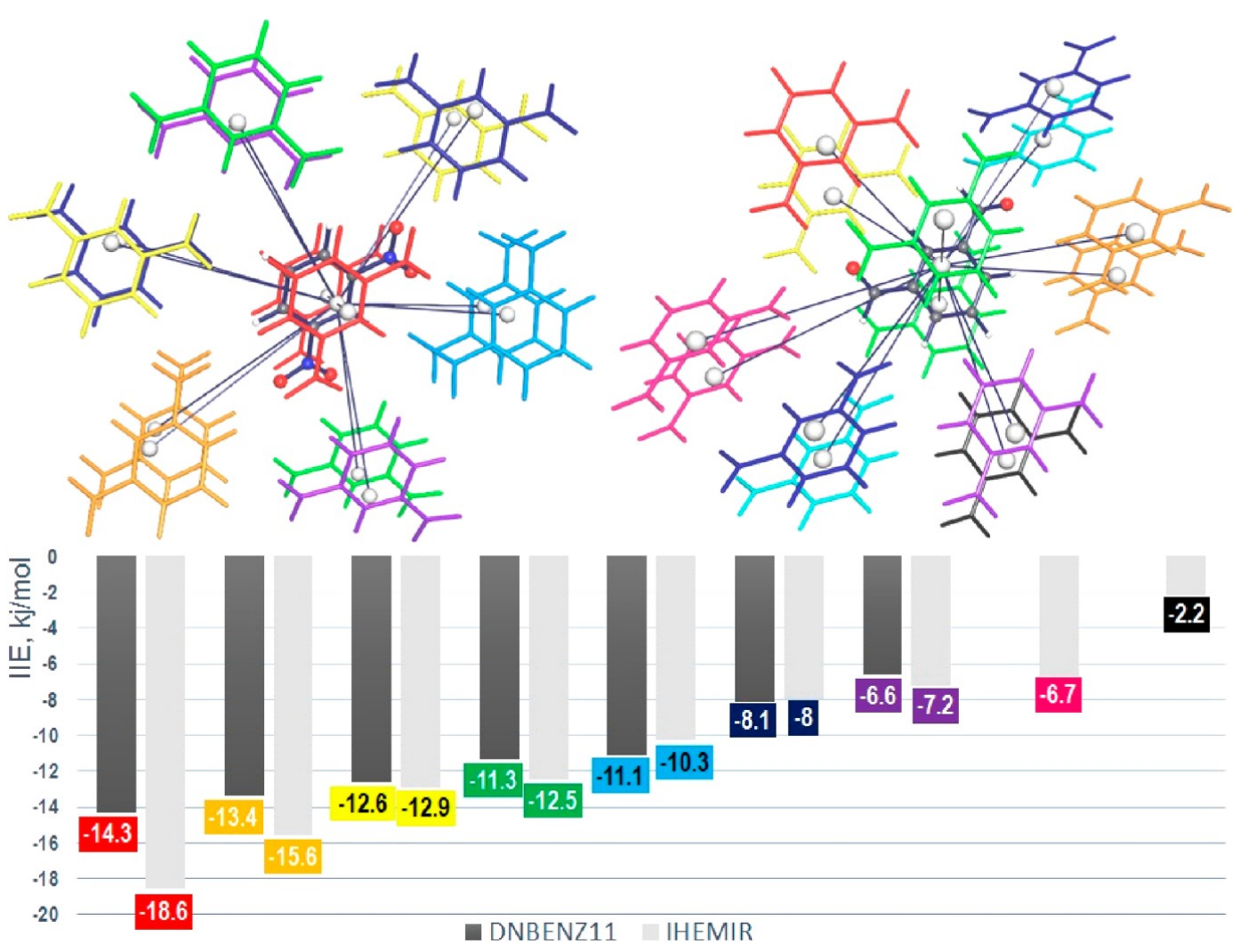

Figure 11. First coordination shell of 1,3-dinitrobenzene molecule (DNBENZ11, left) and benzene-1,3-dicarbaldehyde molecule (IHEMIR, right) in their crystal structures, as well as histogram with IIE in molecular pairs from the first coordination shell. A 14-coordinated net with 14T3 topology corresponds to molecular packings of both structures.

organic crystals the authors ${ }^{57}$ explained it on the basis of "the highly anisotropic nature of the packing" where "strong and weak interaction patterns are present in nearly perpendicular directions"; on the contrary, "... Crystals with comparable intermolecular interactions in all three directions do not show bending and will be hard and brittle, whether the interactions in themselves are strong (hydrogen bonds) or weak (van der Waals)...”. The bending model was proposed based solely on the structural information on 2-methylthionicotinic acid crystal, without any calculation of the interaction energies between molecules in its crystal structure. Using our approach of the overall analysis of intermolecular interaction energies, we reexamined some crystals reported in ref 57 and found out that these requirements do not hold for benzoic acid (brittle) and 1,3-dinitrobenzene (bending) crystals. We calculated the $X$ values for the plane that corresponds to (010) bending faces of 2-methylthionicotinic acid and for plane (001) of benzoic acid showing a similar arrangement of molecules. As seen from Figure 10, both 2 -methylthionicotinic acid (KAMQIZ) ${ }^{58}$ and benzoic acid (BENZAC12) ${ }^{59}$ have almost identical shares of cohesive energy inside double layers of carboxylic acid dimers; quite similar also are the shares of interatomic contacts between these layers. Based on these data, that represent a static picture of the crystal structure, one can say that intermolecular 


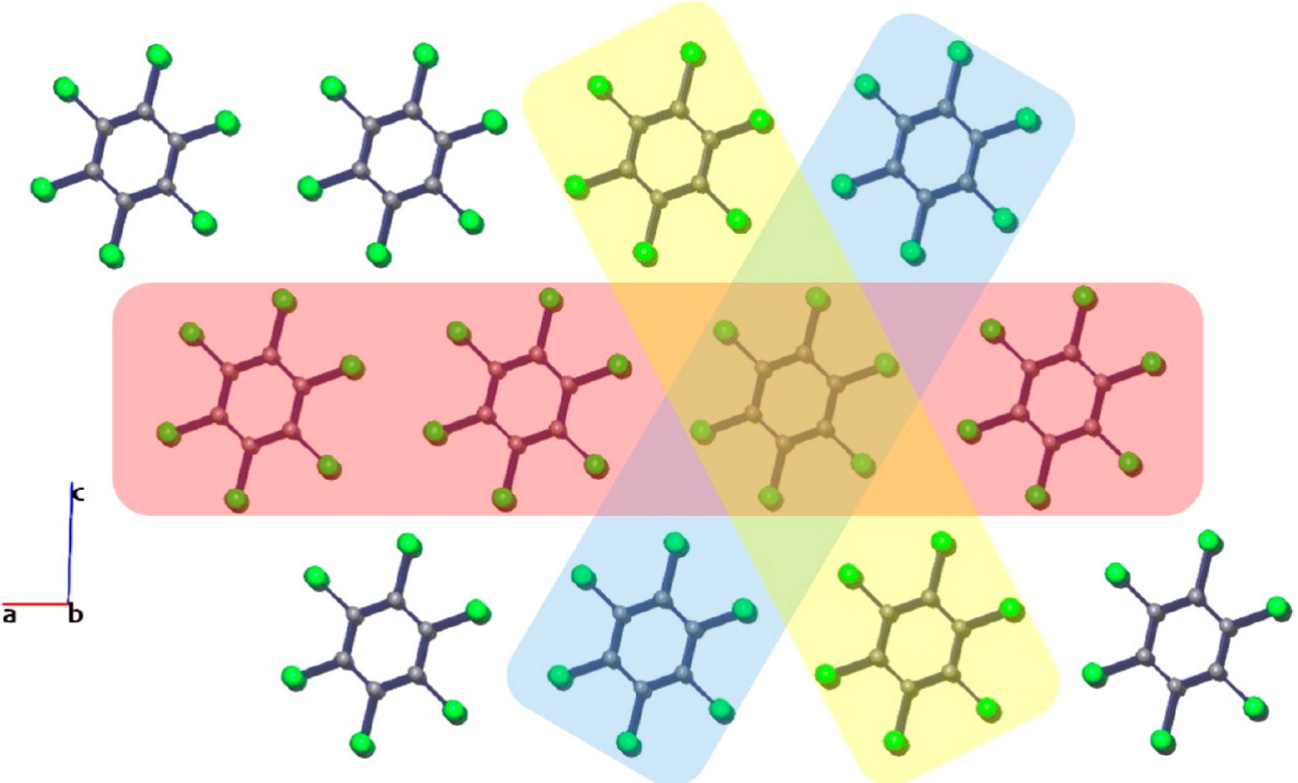

Figure 12. Fragment of the crystal structure of hexachlorobenzene (HCLBNZ11) ${ }^{63}$ projected down [010]. Slices highlighted in red, blue, and yellow

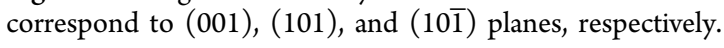

interactions are fairly anisotropic in both structures; moreover, interlayer contacts are alike with $\mathrm{H} \cdots \mathrm{H}$ contacts dominant and these two conditions cannot serve as the only criteria pointing to the possible bending property of the crystal.

The other structure that defies the proposed criteria is metadinitrobenzene (DNBENZ11). ${ }^{60}$ Although the total interaction energies with molecules in the first coordination shell are low and almost identical in all directions, this crystal is reported ${ }^{57}$ to be of the bending type. Conversely, the crystal of benzene-1,3dicarbaldehyde (IHEMIR) ${ }^{61}$ was found to be brittle, ${ }^{57}$ even though it has a similar molecular packing and greater spreading in values of interaction energies (Figure 11). The $X$ values computed for a couple of planes in the structures are the following: DNBENZ11 $X(010)=0.393, X(100)=0.501$, $X(011)=0.338$; IHEMIR $X(10 \overline{1})=0.714, X(101)=0.598$. Low values of $X$ parameters for planes in meta-dinitrobenzene justify the higher isotropy of intermolecular interactions compared to benzene-1,3-dicarbaldehyde crystal.

Naumov and co-workers ${ }^{62}$ conducted a very thorough investigation of the hexachlorobenzene crystal bending processes and all the phenomena that occur within the crystal while under deformation. They found that the origins of this kind of plastic deformation lie in the weak, nonspecific, and "restorable $\mathrm{Cl} \cdots \mathrm{Cl}$ interactions" that allow the sliding of the contracted or expanded (in the inner and outer parts of the kink, respectively) layers, lying in (001) plane, on top of each other. The $X$ value for $(001)$ plane $X(001)=0.638$ is rather small and is almost identical to $X(101)=0.618$ and $X(10 \overline{1})=$ 0.631 ; moreover, interlayer interatomic contacts are the same, i.e., only $\mathrm{Cl} \cdots \mathrm{Cl}$. It shows that the proposed descriptor solely gives no chance to elucidate the found bending of the crystal only on the (001) face (Figure 12).

In ref 22 the authors considered different approaches for identification of slip planes in organic crystals and how these planes affect the mechanical properties of molecular solids. They concluded that the use of attachment energies may be misleading due to the fact that "...the interplanar interaction energy does not capture the essential physics of deformation.
The interplanar interaction energy reflects the work required to detach a layer in a direction perpendicular to the slip plane, while slip by definition is the lateral displacement of the planes relative to each other. This approach therefore fails for interplanar surfaces that are corrugated and which interpenetrate and are difficult to displace" and further "... To capture the essential physics of the problem the characterization of both the detachment energy and the barrier to lateral displacement of crystal planes are required". In our opinion, what is most important for the bending property is a low barrier for sliding of the layers which can be deduced by spike-absent potential energy surface of layers sliding that can be obtained only computationally when the structure is examined in its dynamics. From the structural point of view the indicators of the low sliding barrier might be the presence of nonspecific and weak interlayer interactions or flat interlayer surface or both of them; however, we want to emphasize that only study of the sliding process in its dynamics should be able to distinguish structures that might be plastic.

\section{CONCLUSIONS}

Nowadays, when fast and reliable quantum-chemical computations become available, traditional crystallochemical analysis of crystal structures has to be modified and modernized, since the analyses based on geometry alone, like search for short interatomic contacts, are not enough for a reliable estimation of intermolecular bonding strength. The complexity and variety of intermolecular bonding dictates the necessity to add to conventional analyses the new "energy-based" view on crystal structure. The present study is an example of how helpful the augmentation of topological analysis may be with intermolecular interaction energy calculations. The proposed structure descriptor-the $X$ parameter, whose physical meaning is the share of cohesive energy in a given layer-was used for the interpretation of experimentally observed mechanical properties, namely, cleavage planes in the crystals. The experiments conducted on amino acid crystals revealed that there is a strong correlation between the value of the $X$ parameter for a given 
layer and the observed cleavage planes of those crystals. Integration of this emerging approach with the topological analysis of large crystallographic databases by means of the ToposPro program led to acquisition of a list of perspective organic crystalline substrates for thin film growth by means of OMBE technique. Later on, we can anticipate more objective and automatic search of the planes in the structure with the help of PBC analysis. ${ }^{64-66}$

The ability for extensive plastic deformation, namely bending, is reported for 11 crystals. The bending property is found to be strongly anisotropic - the crystals can be flattened upon themselves when pressure is applied on the bending face; on the contrary, they break when the stress is applied to other faces. In all the crystals the bending face coincides with the cleavage plane, in which the hydrogen-bonded layers with high value of $X$ parameter lie. The analysis of other crystals, reported to be either brittle or ductile in nature, showed that the early explanation of the bending property is improper since it lacks any computational schemes, which could measure the strength of intermolecular interactions. However, one has to admit that the interpretation may be rather complicated and may need the computational simulations of the bending process.

\section{ASSOCIATED CONTENT}

\section{(S) Supporting Information}

The Supporting Information is available free of charge on the ACS Publications website at DOI: 10.1021/acs.cgd.5b01695.

Underlying nets of 199 hydrogen bonded structures Figure S1 and Table S1, Extension of the nomenclature proposed in ref 33, Figure S2 and Table S2 (PDF) Spreadsheet tables with the full classification of the hydrogen bond patterns for 199 structures (X1) and X parameter for 40 structures (X2) (XLSX)

\section{AUTHOR INFORMATION}

\section{Corresponding Authors}

*E-mail massimo.moret@mater.unimib.it.

*E-mail davide.proserpio@unimi.it.

\section{Notes}

The authors declare no competing financial interest.

\section{ACKNOWLEDGMENTS}

P.N.Z. and D.M.P. thank Russian government (grant no. 14.B25.31.0005) and Russian Foundation for Basic Research (grant no. 13-07-00001) for support. P.N.Z. thanks Prof. Vladislav A. Blatov for fruitful discussion of the work. S.R. gratefully acknowledge the generous financial support of Fondazione Cariplo (grant no. 2012-0921). We are grateful to Prof. Angelo Gavezzotti for many fruitful discussions during the stay of P.N.Z. at UNIMI and for providing some changes in his code to be easily interfaced with ToposPro.

\section{REFERENCES}

(1) Campione, M.; Sassella, A.; Moret, M.; Papagni, A.; Trabattoni, S.; Resel, R.; Lengyel, O.; Marcon, V.; Raos, G. J. Am. Chem. Soc. 2006, 128, 13378-13387.

(2) Haber, T.; Resel, R.; Thierry, A.; Campione, M.; Sassella, A.; Moret, M. Phys. E 2008, 41, 133-137.

(3) Campione, M.; Raimondo, L.; Moret, M.; Campiglio, P.; Fumagalli, E.; Sassella, A. Chem. Mater. 2009, 21, 4859-4867.

(4) Raimondo, L.; Moret, M.; Campione, M.; Borghesi, A.; Sassella, A. J. Phys. Chem. C 2011, 115, 5880-5885.
(5) Raimondo, L.; Fumagalli, E.; Moret, M.; Campione, M.; Borghesi, A.; Sassella, A. J. J. Phys. Chem. C 2013, 117, 13981-13988.

(6) Trabattoni, S.; Moret, M.; Campione, M.; Raimondo, L.; Sassella, A. Cryst. Growth Des. 2013, 13, 4268-4278.

(7) Trabattoni, S.; Raimondo, L.; Campione, M.; Braga, D.; Holmberg, V. C.; Norris, D. J.; Moret, M.; Ciavatti, A.; Fraboni, B.; Sassella, A. Adv. Mater. Interfaces 2015, 2, 1500423.

(8) Kaviyil, S. E.; Campione, M.; Sassella, A.; Su, G. B.; He, Y. P.; Chen, C. J.; Borghesi, A. J. Chem. Phys. 2012, 137, 224703.

(9) Sitter, H.; Andreev, A.; Matt, G.; Sariciftci, S. N. Synth. Met. 2003, 138, 9-13.

(10) Allen, F. H. Acta Crystallogr., Sect. B: Struct. Sci. 2002, B58, 380388.

(11) Blatov, V. A.; Shevchenko, A. P.; Proserpio, D. M. Cryst. Growth Des. 2014, 14, 3576-3586.

(12) Gavezzotti, A. J. Phys. Chem. B 2002, 106, 4145-4154. Gavezzotti, A. J. Phys. Chem. B 2003, 107, 2344-2353.

(13) Gavezzotti, A. New J. Chem. 2011, 35, 1360-1368.

(14) Bandyopadhyay, R.; Grant, D. J. W. Pharm. Res. 2002, 19, 491496.

(15) Fries, D. C.; Rao, S. T.; Sundaralingam, M. Acta Crystallogr., Sect. B: Struct. Crystallogr. Cryst. Chem. 1971, B27, 994-1005.

(16) Wittmann, J.-C.; Lotz, B. J. J. Polym. Sci., Polym. Phys. Ed. 1981, $19,1837-1851$

(17) Wittmann, J.-C.; Hodge, A. M.; Lotz, B. J. J. Polym. Sci., Polym. Phys. Ed. 1983, 21, 2495-2509.

(18) Parikh, D.; Phillips, P.J. J. J. Polym. Sci., Polym. Lett. Ed. 1990, 28, $167-172$.

(19) De Rosa, C.; Park, C.; Lotz, B.; Wittmann, J.-C.; Fetters, L.-J.; Thomas, E. L. Macromolecules 2000, 33, 4871-4876.

(20) Brinkmann, M.; Contal, C.; Kayunkid, N.; Djuric, T.; Resel, R. Macromolecules 2010, 43, 7604-7610.

(21) Li, H.; Yan, S. Macromolecules 2011, 44, 417-428.

(22) Shariare, M. H.; Leusen, F. J. J.; de Matas, M.; York, P.; Anwar, J. Pharm. Res. 2012, 29, 319-331.

(23) Peresypkina, E. V.; Blatov, V. A. Acta Crystallogr., Sect. B: Struct. Sci. 2000, B56, 1035-1045.

(24) Baburin, I. A.; Blatov, V. A. Acta Crystallogr., Sect. B: Struct. Sci. 2007, B63, 791-802.

(25) O'Keeffe, M.; Peskov, M. A.; Ramsden, S. J.; Yaghi, O. M. Acc. Chem. Res. 2008, 41, 1782-1789.

(26) Koch, E.; Fischer, W. Z. Kristallogr. 1978, 148, 107-152.

(27) Alexandrov, E. V.; Blatov, V. A.; Kochetkov, A. V.; Proserpio, D. M. CrystEngComm 2011, 13, 3947-3958.

(28) Aman, F.; Asiri, A. M.; Siddiqui, W. A.; Arshad, M. N.; Ashraf, A.; Zakharov, N. S.; Blatov, V. A. CrystEngComm 2014, 16, 19631970.

(29) Papavinasam, E.; Natarajan, S.; Shivaprakash, N. C. Int. J. Pept. Protein Res. 1986, 28, 525-528.

(30) Frisch, M. J.; Trucks, G. W.; Schlegel, H. B.; Scuseria, G. E.;Robb, M. A.; Cheeseman, J. R.; Scalmani, G.; Barone, V.; Mennucci, B.; Petersson, G. A.; Nakatsuji, H.; Caricato, M.; Li, X.; Hratchian, H. P.; Izmaylov, A. F.; Bloino, J.; Zheng, G.; Sonnenberg, J. L.; Hada, M.; Ehara, M.; Toyota, K.; Fukuda, R.; Hasegawa, J.; Ishida, M.; Nakajima, T.; Honda, Y.; Kitao, O.; Nakai, H.; Vreven, T.; Montgomery, J. A., Jr.; Peralta, J. E.; Ogliaro, F.; Bearpark, M.; Heyd, J. J.; Brothers, E.; Kudin, K. N.; Staroverov, V. N.; Kobayashi, R.; Normand, J.; Raghavachari, K.; Rendell, A.; Burant, J. C.; Iyengar, S. S.; Tomasi, J.; Cossi, M.; Rega,N.; Millam, J. M.; Klene, M.; Knox, J. E.; Cross, J. B.; Bakken, V.; Adamo, C.; Jaramillo, J.; Gomperts, R.; Stratmann, R. E.; Yazyev, O.; Austin, A. J.; Cammi, R.; Pomelli, C.; Ochterski, J. W.; Martin, R. L.; Morokuma, K.; Zakrzewski, V. G.; Voth, G. A.; Salvador, P.; Dannenberg, J. J.; Dapprich, S.; Daniels, A. D.; Farkas, Ö.; Foresman, J. B.; Ortiz, J. V.; Cioslowski, J.; Fox, D. J. Gaussian 09, Revision A.1; Gaussian, Inc.: Wallingford, CT, 2009.

(31) Maschio, L.; Civalleri, B.; Ugliengo, P.; Gavezzotti, A. J. Phys. Chem. A 2011, 115, 11179-11186.

(32) Bruker APEX2 Software Package; Bruker AXS Inc.: Madison, Wisconsin, USA. 
(33) Görbitz, C. H.; Vestli, K.; Orlando, R. Acta Crystallogr., Sect. B: Struct. Sci. 2009, B65, 393-400.

(34) van de Streek, J.; Neumann, M. A. Acta Crystallogr., Sect. B: Struct. Sci., Cryst. Eng. Mater. 2014, B70, 1020-1032.

(35) Dunitz, J. D.; Gavezzotti, A. Cryst. Growth Des. 2012, 12, 58735877.

(36) Sun, C. C.; Kiang, Y.-H. J. Pharm. Sci. 2008, 97, 3456-3461.

(37) Gilli, G.; Gilli, P. The Nature of the Hydrogen Bond; University

Press: Oxford, 2009.

(38) Demartin, F.; Filippini, G.; Gavezzotti, A.; Rizzato, S. Acta Crystallogr., Sect. B: Struct. Sci. 2004, B60, 609-620.

(39) Gavezzotti, A. Struct. Chem. 2005, 16, 177-185.

(40) Hoepfner, V.; Deringer, V. L.; Dronskowski, R. J. Phys. Chem. A 2012, 116, 4551-4559.

(41) George, J.; Deringer, V. L.; Dronskowski, R. Inorg. Chem. 2015, 54, 956-962.

(42) Deringer, V. L.; Pan, F.; George, J.; Müller, P.; Dronskowski, R.; Englert, U. CrystEngComm 2014, 16, 135-138.

(43) Dunitz, J. D.; Gavezzotti, A. Angew. Chem., Int. Ed. 2005, 44, $1766-1787$.

(44) Shishkin, O. V.; Zubatyuk, R. I.; Shishkina, S. V.; Dyakonenko, V. V.; Medviediev, V. V. Phys. Chem. Chem. Phys. 2014, 16, 67736786.

(45) Turner, M. J.; Thomas, S. P.; Shi, M. W.; Jayatilaka, D.; Spackman, M. A. Chem. Commun. 2015, 51, 3735-3738.

(46) Borc, J.; Sangwal, K. Surf. Sci. 2008, 602, 1175-1184.

(47) Borc, J.; Sangwal, K. Surf. Sci. 2007, 601, 1160-1166.

(48) Gidalevitz, D.; Feidenhans'l, R.; Matlis, S.; Smilgies, D.-M.; Christensen, M. J.; Leiserowitz, L. Angew. Chem., Int. Ed. Engl. 1997, 36, 955-959.

(49) Dalhus, B.; Görbitz, C. H. Acta Crystallogr., Sect. C: Cryst. Struct. Commun. 1996, C52, 1759-1761.

(50) Dalhus, B.; Görbitz, C. H.; et al. Acta Chem. Scand. 1996, 50, 544-548.

(51) Dalhus, B.; Görbitz, C. H. Acta Crystallogr., Sect. C: Cryst. Struct. Commun. 1999, C55, 1547-1555.

(52) Dalhus, B.; Görbitz, C. H. Acta Crystallogr. 1999, C55, 1

DOI: $10.1107 /$ S0108270199099291.

(53) Dalhus, B.; Görbitz, C. H. Acta Crystallogr., Sect. B: Struct. Sci. 1999, B55, 424-431.

(54) Dalhus, B.; Görbitz, C. H. Acta Crystallogr., Sect. B: Struct. Sci. 2000, B56, 720-727.

(55) Görbitz, C. H.; Rissanen, K.; Valkonen, A.; Husabo, A. Acta Crystallogr., Sect. C: Cryst. Struct. Commun. 2009, C65, o267-o272.

(56) Arkhipov, S. G.; Rychkov, D. A.; Pugachev, A. M.; Boldyreva, E. V. Acta Crystallogr., Sect. C: Struct. Chem. 2015, C71, 584-592.

(57) Reddy, C. M.; Padmanabhan, K. A.; Desiraju, G. R. Cryst. Growth Des. 2006, 6, 2720-2731.

(58) Basavoju, S.; Reddy, C. M.; Desiraju, G. R. Acta Crystallogr., Sect.

E: Struct. Rep. Online 2005, E61, o822-o823.

(59) Nieger, M. Private Communication, 2010 CCDC 787428.

(60) Wojcik, G.; Mossakowska, I.; Holband, J.; Bartkowiak, W. Acta Crystallogr., Sect. B: Struct. Sci. 2002, B58, 998-1004.

(61) Britton, D. Acta Crystallogr., Sect. E: Struct. Rep. Online 2002, C58, o637-o639.

(62) Panda, M. K.; Ghosh, S.; Yasuda, N.; Moriwaki, T.; Mukherjee, G. D.; Reddy, C. M.; Naumov, P. Nat. Chem. 2014, 7, 65-72.

(63) Brown, G. M.; Strydom, O. A. W. Acta Crystallogr., Sect. B: Struct. Crystallogr. Cryst. Chem. 1974, B30, 801-804.

(64) Hartman, P.; Perdok, W. G. Acta Crystallogr. 1955, 8, 49-52.

(65) Hartman, P.; Perdok, W. G. Acta Crystallogr. 1955, 8, 521-524.

(66) Hartman, P.; Perdok, W. G. Acta Crystallogr. 1955, 8, 525-539. 